\title{
Imaging with hypertelescopes: a simple modal approach
}

\author{
C. Aime \\ Université de Nice Sophia Antipolis, Centre National de la Recherche Scientifique, UMR 6525 H. Fizeau, Observatoire de la Côte \\ d'Azur, Campus Valrose, 06108 Nice Cedex 2, France \\ e-mail: Claude. Aime@unice.fr
}

Received 12 September 2007 / Accepted 18 February 2008

\section{ABSTRACT}

\begin{abstract}
Aims. We give a simple analysis of imaging with hypertelescopes, a technique proposed by Labeyrie to produce snapshot images using arrays of telescopes. The approach is modal: we describe the transformations induced by the densification onto a sinusoidal decomposition of the focal image instead of the usual point spread function approach.

Methods. We first express the image formed at the focus of a diluted array of apertures as the product $R_{0}(\alpha) X_{\mathrm{F}}(\alpha)$ of the diffraction pattern of the elementary apertures $R_{0}(\alpha)$ by the object-dependent interference term $X_{\mathrm{F}}(\alpha)$ between all apertures. The interference term, which can be written in the form of a Fourier Series for an extremely diluted array, produces replications of the object, which makes observing the image difficult. We express the focal image after the densification using the approach of Tallon and Tallon-Bosc. Results. The result is very simple for an extremely diluted array. We show that the focal image in a periscopic densification of the array can be written as $R_{0}(\alpha) X_{\mathrm{F}}(\alpha / \gamma)$, where $\gamma$ is the factor of densification. There is a dilatation of the interference term while the diffraction term is unchanged. After de-zooming, the image can be written as $\gamma^{2} X_{\mathrm{F}}(\alpha) R_{0}(\gamma \alpha)$, an expression which clearly indicates that the final image corresponds to the center of the Fizeau image intensified by $\gamma^{2}$. The imaging limitations of hypertelescopes are therefore those of the original configuration. The effect of the suppression of image replications is illustrated in a numerical simulation for a fully redundant configuration and a non-redundant one.
\end{abstract}

Key words. instrumentation: interferometers - instrumentation: high angular resolution - telescopes

\section{Introduction}

Labeyrie (1996) proposed to obtain snapshot images from a diluted array of telescopes by rearranging the output pupil using the periscopic principle of Michelson. The technique, called hypertelescope or densified aperture, is restricted to conformal transformations in which the pattern of sub-pupil centers is identical in the entrance and exit pupils. One of the particularities of the technique is that the point spread function is no longer space invariant, because the interference and diffraction patterns of the telescopes move at different velocities according to the position of the point source on the sky. This makes it difficult to derive the final image. Several recent publications were made on this subject, especially concerning the field of view, as discussed by Lardière et al. (2007) and Patru et al. (2007).

The analysis presented here is modal. It does not use the space-varying point spread function, but analyzes the transformations produced by a hypertelescope on the angular frequencies transmitted by the array. For that we use the results of Tallon \& Tallon-Bosc (1992), who compared Fizeau and Michelson interferometers. We will give a simple formulation for the case, already considered by Labeyrie (1996), of an highly diluted array and a very small object.

\section{Imaging with telescopes arrays}

\subsection{Basic formulation of image formation}

For the usual mode of operation of telescopes, the image $I(\alpha)$ in the focal plane is given by the convolution relation:

$I(\alpha)=R(\alpha) * O(\alpha)$, where $*$ denotes the $2 \mathrm{D}$ convolution on components $\left(\alpha_{x}, \alpha_{y}\right)$ of $\alpha$, the angular coordinates on the sky. $O(\alpha)$ is the geometrical image of the object in the focal plane, and $R(\alpha)$ is the Point Spread Function (PSF), given by:

$R(\alpha)=\frac{1}{S \lambda^{2}}\left|\hat{P}\left(\frac{\alpha}{\lambda}\right)\right|^{2}$

where $\lambda$ is the light wavelength and $S$ is the area of the telescope aperture of transmission $P(r)$, with $r=(x, y)$. In the Fourier plane, the object image relationship appears as a linear filtering:

$\widehat{I}(u)=T(u) \times \widehat{O}(u)$,

where $u$ denotes $2 \mathrm{D}$ angular frequencies $\left(u_{x}, u_{y}\right), \widehat{O}(u)$ is the image transform, and $T(u)$ is the Optical Transfer Function (OTF), a complex function that describes the transmission in amplitude and phase of the angular frequencies. Taking the Fourier transform of Eq. (2), the OTF can be written as the aperture autocorrelation function:

$T(u)=\frac{1}{S} \int P(r) P^{*}(r-\lambda u) \mathrm{d} r$.

These relations are valid regardless of the fact that $P(r)$ is a monolithic aperture or a collection of diluted apertures. For $K$ (non-overlapping) identical apertures of transmissions $P_{0}(r)$, centered at $\left\{r_{k}\right\}$, the transmission $P(r)$ can be written as:

$P(r)=P_{0}(r) * \sum_{k=1}^{K} \delta\left(r-r_{k}\right)$, 
and the corresponding PSF takes the form:

$R(\alpha)=\frac{1}{S \lambda^{2}}\left|\hat{P}_{0}\left(\frac{\alpha}{\lambda}\right)\right|^{2}\left|\sum_{k=1}^{K} \exp \left(-\frac{2 i \pi \alpha r_{k}}{\lambda}\right)\right|^{2}$,

where $S=K S_{0}, S_{0}$ being the area of an elementary aperture. The corresponding OTF can be written as:

$T(u)=T_{0}(u)+\frac{1}{K} \sum_{l=1}^{K} \sum_{k \neq l}^{K} T_{0}\left(u-\frac{r_{l}-r_{k}}{\lambda}\right)$,

which acts as a multiple band-pass linear filter that transmits the angular frequencies in discrete zones of the frequency plane.

\subsection{Imaging in the Fizeau mode}

The imaging of stellar or planetary surfaces will require resolutions corresponding to arrays spreading over tens of kilometers. The resulting array will be extremely diluted and the surface $S$ of each individual telescope will be negligible compared to the dimensions of the array. Each individual telescope will not resolve the object and therefore there will be no measurable variation of $\hat{O}(u)$ over $T_{0}(u)$. We can then use the simplified expression:

$\hat{I}(u)=T(u) \hat{O}(u) \sim T_{0}(u)+\frac{1}{K} \sum_{l=1}^{K} \sum_{k \neq l}^{K} A_{l k} \exp \left(i \varphi_{l k}\right) T_{0}\left(u-d_{l k}\right)$

where $d_{l k}=\left(r_{l}-r_{k}\right) / \lambda$, and $A_{l k}$ and $\varphi_{l k}$ are the amplitude and phase of $\hat{O}\left(d_{l k}\right)$. The focal plane image can be written:

$$
\begin{aligned}
I_{\mathrm{F}}(\alpha) & =\left(1+\frac{1}{K} \sum_{l=1}^{K} \sum_{k \neq l}^{K} A_{l k} \cos \left(2 \pi d_{l k} \alpha-\varphi_{l k}\right)\right) R_{0}(\alpha) \\
& =X_{\mathrm{F}}(\alpha) R_{0}(\alpha)
\end{aligned}
$$

where $R_{0}(\alpha)$ is the envelop of the diffraction pattern of the elementary apertures and $X_{\mathrm{F}}(\alpha)$ is the interference term of the form a Fourier Series whose coefficients depend on the object.

\subsection{Imaging in the Michelson mode: hypertelescopes}

The principle of hypertelescopes is described in Labeyrie (1996). The array densification may be obtained extending to many sub-apertures the periscopic arrangement of Michelson's 20 -feet interferometer. Alternatively it is possible to use inverted Galilean telescopes to increase the diameter of the elementary apertures (Labeyrie 2002). The two techniques may be used simultaneously and Labeyrie (1996) defines a pupil concentration coefficient $\gamma$ that takes into account the two aspects. The result will be the same regardless of the technique used, but the mathematical formalism to describe the densification operation in terms of imaging is different in the intermediate results. For example the use of Galilean telescopes imposes a reduction of the wave amplitude to keep the energy constant, which is not the case for the Michelson periscopic arrangement we consider here. In accordance with Labeyrie's convention, $\gamma$ is the densification parameter (greater than 1) equal to the ratio of telescope center separations before and after the densifying operation.

The mathematical formalism necessary to describe hypertelescopes was given four years before Labeyrie (1996)'s paper by Tallon \& Tallon-Bosc (1992). They showed that the Michelson periscopic system performs a change of angular frequencies in the image. The part of the Fourier plane transmitted by a pair of telescopes around the frequency $u=d / \lambda$ is shifted as a whole to a lower frequency $u^{\prime}=u / \gamma$, without any modification of the frequency transmission in modulus and phase. This result was obtained using subtle manipulations of Fourier optics. Because of these frequency translations, the system is no longer a linear system and there is no reason to look for a relation of convolution in the usual sense of the term. On the contrary, a spacevarying point spread response is required to perform the frequency shifting.

For extremely diluted arrays, the individual apertures can only sample the angular frequency plane in a collection of pointlike regions. Then the translation operation merges with a simple homothetic transformation of a factor $\gamma$. In that case, it is possible to give a very simple demonstration of Tallon and Tallon-Bosc result, as we show in the Appendix.

Applying Tallon and Tallon-Bosc's result to the case of the extremely diluted array corresponds to substitute $d_{l k}^{\prime}=d_{l k} / \gamma$ to $d_{l k}$ in Eq. (9). Denoting $I_{M}(\alpha)$ the observed focal plane image after densification, we can write:

$$
\begin{aligned}
I_{M}(\alpha) & =\left(1+\frac{1}{K} \sum_{l=1}^{K} \sum_{k \neq l}^{K} A_{l k} \cos \left(2 \pi \frac{d_{l k}}{\gamma} \alpha-\varphi_{l k}\right)\right) R_{0}(\alpha) \\
& =X_{M}(\alpha) R_{0}(\alpha)=X_{\mathrm{F}}\left(\frac{\alpha}{\gamma}\right) R_{0}(\alpha)
\end{aligned}
$$

The interferometric term $X_{\mathrm{F}}(\alpha / \gamma)$ is dilated by the factor $\gamma$, while the diffraction term is unchanged. In a heuristic manner, the result can be described as follows: we observe in the focal plane the interferometric object-dependent image $X_{\mathrm{F}}(\alpha / \gamma)$ through a fixed window $R_{0}(\alpha)$. As the rate of densification $\gamma$ is increased, the interferometric pattern is zoomed while the observed region remains unchanged. In the limiting case, only the central part of the image is left, therefore corresponding to a snapshot usable picture, as explained by Labeyrie (1996). In Eq. (10) there is no explicit amplification of the image intensity, and just a dilatation of its size by a factor $\gamma$. The two effects are however equivalent. The amplification appears if we keep the image size unchanged on the sky by de-zooming the whole picture of the factor $\gamma$. The flux is unchanged, and we have:

$I_{M}(\gamma \alpha)=\gamma^{2} X_{\mathrm{F}}(\alpha) R_{0}(\gamma \alpha)$

which shows that the resulting image corresponds to the Fizeau image limited to its center and intensified by the term $\gamma^{2}$. In the limiting case, $R_{0}(\gamma \alpha)$ restricts the image to a single object, without replications. The gain $\gamma^{2}$ corresponds to the number of object replications in the Fizeau image that are summed in the densified image. The hypertelescope works as an optical device that performs an analog addition of all individual images replicated by the Fizeau array. Equation (11) also shows that the conditions of the field of view are the same in a hypertelescope as the original array in the Fizeau configuration. That conclusion was already indicated, but not really demonstrated, in several papers (Lardiere 2007; Patru 2007).

Note that Eqs. (10) and (11) are consistent with Labeyrie (1996)'s results. In Eq. (3) of this paper, the intensity in the image is first written as a Fredholm equation (called a pseudoconvolution). An approximate expression is derived for $I_{M}(\alpha)$. It is denoted $B(x, y)$ in Eq. (4). It can be rewritten as:

$I_{M}(\alpha) \approx \frac{1}{\gamma^{2}} R_{0}(\alpha)\left[O\left(\frac{\alpha}{\gamma}\right) \otimes \operatorname{Int}(\alpha)\right]$

where $\operatorname{Int}(\alpha)$ corresponds to the interference function of the densified array (called $I(x, y)$ in Labeyrie's paper). In this form the 
interest of hypertelescopes is not obvious, especially for the term $\gamma^{-2}$ that seems to reduce the image intensity. However, if we apply the same de-zooming procedure as discussed above, Eq. (12) becomes:

$I_{M}(\gamma \alpha) \approx \gamma^{2} R_{0}(\gamma \alpha)[O(\alpha) \otimes \operatorname{Int}(\gamma \alpha)]$

where we have made use of the fact that for a two-dimensional convolution, if $h(\alpha)=f(\alpha) \otimes g(\alpha)$, then $h(\gamma \alpha)=\gamma^{2} f(\gamma \alpha) \otimes g(\gamma \alpha)$. This formulation shows that the present formal approach is fully compatible with Labeyrie's more intuitive results.

A numerical illustration is given in the next section for a better understanding of the densified aperture technique.

\section{Numerical illustrations}

The shape of $X_{\mathrm{F}}(\alpha)$ strongly depends on the array configuration. Its expression is not simple, in general. It takes a convenient form for a redundant array of telescopes equally spaced by a distance $d$ on a regular square grid at $K=N \times N$ positions. The OTF covers $(2 N-1)^{2}$ regions of the frequency plane on a regular grid, of the form:

$$
\begin{aligned}
T\left(u_{x}, u_{y}\right)= & \sum_{p=1-N}^{N-1} \sum_{q=1-N}^{N-1} \frac{(N-|p|)(N-|q|)}{N^{2}} \\
& \times T_{0}\left(u_{x}-p \frac{d}{\lambda}, u_{y}-q \frac{d}{\lambda}\right),
\end{aligned}
$$

and the PSF simplifies to:

$$
\begin{aligned}
R\left(\alpha_{x}, \alpha_{y}\right)= & \frac{1}{S \lambda^{2}}\left|\hat{P}_{0}\left(\frac{\alpha_{x}}{\lambda}, \frac{\alpha_{y}}{\lambda}\right)\right|^{2} \\
& \times\left(\frac{\sin \left(N \pi d \alpha_{x} / \lambda\right)}{\sin \left(\pi d \alpha_{x} / \lambda\right)} \frac{\sin \left(N \pi d \alpha_{y} / \lambda\right)}{\sin \left(\pi d \alpha_{y} / \lambda\right)}\right)^{2} .
\end{aligned}
$$

These expressions are those of a two-dimensional grating. The PSF presents peaks of width $\lambda /(N d)$, each $\lambda / d$. The overall spatial extension is given by the diffraction pattern of the elementary apertures. For circular apertures of diameter $D$, the number of peaks is of the order of $(d / D)^{2}$. The convolution between the object and the PSF produces replications. To avoid overlapping of the images, the object extent cannot be larger than $\lambda / d$.

We give in Fig. 1 an illustration of the effect of aperture densification for the observation of a Saturn like exoplanet using a fully redundant array of 49 apertures set on a square grid $(7 \times 7)$. The elementary apertures are squares of side length 3 units and the distances between telescopes centers are 20 units (arbitrary units). The figure shows the results for three operating modes, the direct Fizeau configuration and two rates of densification. One rate corresponds to the complete aperture densification where the images of the elementary apertures are brought close-by to form a monolithic aperture, as in the original Labeyrie (1996) proposal. In the other rate of densification, that we may call OTF densification, a distance is left between apertures so that there is no overlapping of individual autocorrelation functions of elementary telescopes and no overlapping of frequencies in the Fourier plane.

For the numerical computation, we have used the complete Tallon \& Tallon-Bosc (1992) model to synthesize the resulting image, without any approximation. The operation is performed in the Fourier plane. The array of $N \times N$ apertures, with $N=7$, gives the object's spectrum for $(2 N-1)^{2}=169$ regions of the Fourier plane, as shown in Fig. 1 for the modulus of the image


Fig. 1. Numerical illustrations. Left three figures: Fizeau imaging. Top: the array of $7 \times 7$ square apertures (side length 3 units, separation between aperture centers of 20 units); below: the object angular frequencies in the Fourier (modulus in a log scale); bottom: the direct image showing the object replication. Center three figures, from top to bottom: densified aperture for a separation reduced to 5 units, the Fourier plane showing the non-overlapping densified OTF (only the central non-zero part is shown) and the focal image. Right three figures: same as center for a full densification. The focal plane images are drawn at the same scale, and the zooming effect of Eq. (10) appears clearly.

transform. The first step in the procedure leading to the densified image consists of translating the high frequency parts towards the lower frequencies, each of the 168 external regions being moved towards the low frequencies. The result for the OTF densification is a low frequency padding of the Fourier plane, without any frequency overlapping, while the complete aperture densification induces a marginal frequency overlapping. At least for the object we have chosen, it seems that this frequency overlap will not produce visible modification in the final image.

Final focal plane images are obtained by a Fourier transform of the densified Fourier plane. The approach is modal in the sense that we have considered only angular frequencies in the Fourier plane. There is no need in this approach to compute the space varying response for each point source to obtain the final image. The resulting image is obtained exactly and very quickly, without the need to integrate the Fredholm relation. The resulting images of this exact simulation are in good agreement with the approximated expression of Eq. (10). The zooming effect on the object is clearly visible.

In the illustration of Fig. 1, the distances between telescope centers were chosen so as to correctly sample the object's Fourier transform. We illustrate in Fig. 2 what happens with the same array if separations between apertures are increased to be too large to correctly sample the object's Fourier transform. This undersampling in the frequency plane produces an aliasing in the direct plane. The object images overlap and the observation becomes useless.

This simulation illustrates the fact that the image properties are fully defined by the geometry of the original array, and do not depend on the mode of configuration of the exit aperture. A very important point is that the object's Fourier plane must be correctly sampled by the array configuration. Obtaining resolved information within each individual region $T_{0}\left(u-d_{l k}\right)$ is useless in terms of the Shannon theorem. 

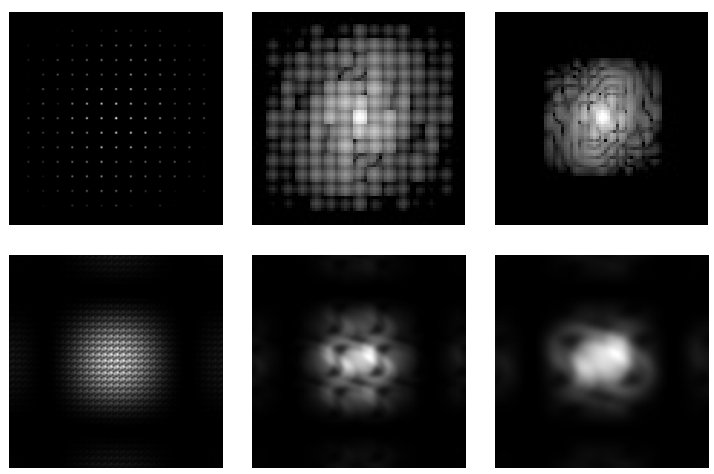

Fig. 2. Same array as in Fig. 1, but for a distance between apertures enlarged to 35. Left: Fizeau imaging showing the object replications. Center: densified apertures corresponding to a distance of 5 between apertures. Right: close-by densification 3. The overlapping of images is present in the Fizeau configuration and the image cannot be recovered.
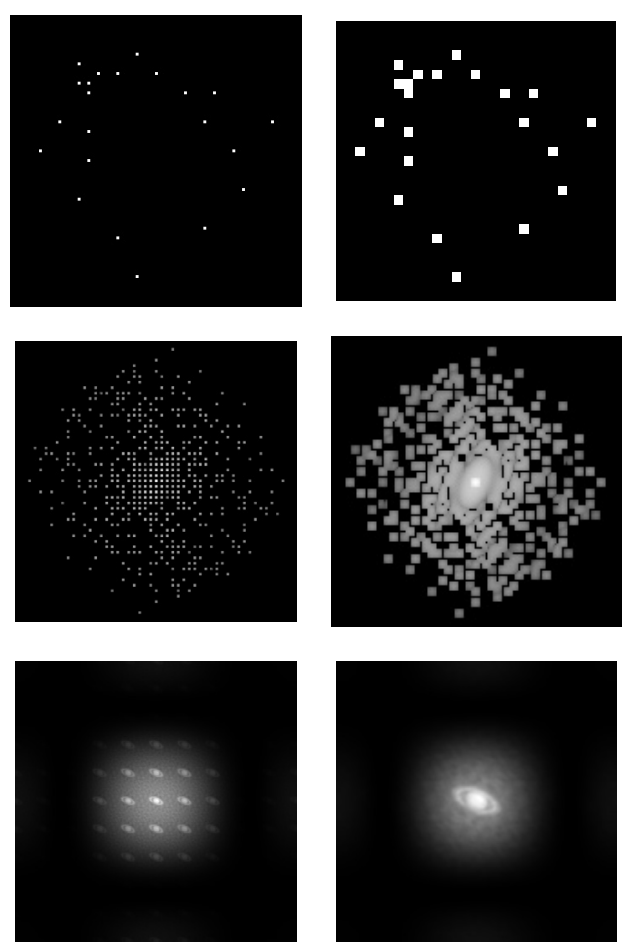

Fig. 3. Example of the result that can be obtained with a fully nonredundant array of 22 square apertures (side length 3 units, minimum distance between aperture centers 10 units). Left: Fizeau imaging. Right: hypertelescope configuration. From top to bottom: aperture, Fourier and focal plane.

A fully redundant two-dimensional apertures is certainly not the best solution. In terms of frequency coverage, non redundant configurations are more efficient. We give in Fig. 3 an example of what could be obtained for an array of 22 non-redundant apertures giving the object's spectrum for 463 regions of the Fourier plane. In that simulation, that uses the same elementary square apertures as before, the minimum distance between apertures is 10 units. The maximum densification is obtained when the closest apertures are joined. This effect is clearly visible for the three elementary apertures in the upper left part of the array (Fig. 3 top-right). However the densified non-redundant aperture remains rather sparse, and is no longer similar to a monolithic aperture. What is densified is the OTF, at least for its central part there. Note that the configuration shown here is for illustration only. Finding the optimal configuration for a given number of apertures is not treated here. The images shown here are raw results, with no attempt to improve the resolution by any image processing. In that respect, there is probably much more to expect from the data processing of non-redundant arrays, especially if a technique can be used to fill in the frequency gaps left by these configurations.

Acknowledgements. The author would like to thank the referee Isabelle Tallon-Bosc for very constructive comments.

\section{Appendix A: A simplified presentation of aperture densification}

The approach developed by Tallon \& Tallon-Bosc (1992) is complete, and there is no need to further develop their computation. However, for a better understanding of the technique, we present a simplified heuristic presentation in the case of extremely diluted arrays.

We describe it for a system of two telescopes. A point source of the object at the angular position $\xi$ produces on the aperture plane a wavefront tilted by the angle $\xi$. The path difference between the two aperture is $\xi d$. Orientating the axis in the focal plane as on the sky, it produces a fringe pattern of the form $\cos (2 \pi(\alpha d-\xi d) / \lambda)$ for the oscillatory term. Now let us consider the object as a sum of sinusoidal terms such as $A(v) \cos (2 \pi v \xi-\varphi(v))$. Summing the point responses for all positions $\xi$, we reach the well known result:

$$
\begin{aligned}
S_{\mathrm{F}}(\alpha) & =A(v) \int \cos (2 \pi v \xi-\varphi(v)) \cos (2 \pi(\alpha d-\xi d) / \lambda) \mathrm{d} \xi \\
& =A\left(\frac{d}{\lambda}\right) \cos \left(2 \pi \frac{\alpha d}{\lambda}-\varphi\left(\frac{d}{\lambda}\right)\right) .
\end{aligned}
$$

When the exit aperture is densified from $d$ to $d^{\prime}$, the fringe pattern in the focal plane has a frequency $d^{\prime} / \lambda$. The path difference between apertures remains $\xi d$, and the telescope response to a point source in the direction $\xi$ becomes $\cos \left(2 \pi\left(\alpha d^{\prime}-\xi d\right) / \lambda\right)$. Substituting this quantity to $\cos (2 \pi(\alpha d-\xi d) / \lambda)$ in the above equation, we directly obtain the result:

$S_{M}(\alpha)=A\left(\frac{d}{\lambda}\right) \cos \left(2 \pi \frac{\alpha d^{\prime}}{\lambda}-\varphi\left(\frac{d}{\lambda}\right)\right)=S_{\mathrm{F}}\left(\frac{\alpha}{\gamma}\right)$

which demonstrates the rule used to derive Eq. (10).

\section{References}

Labeyrie, A. 1996, A\&A, 118, 517

Labeyrie, A. 2002, EAS Pub. Ser., 8, 327

Lardière, O., Martinache, F., \& Patru, F. 2007, MNRAS, 375, 977

Patru, F., Mourard, D., Lardière, O., \& Lagarde, S. 2007, MNRAS, 376, 1047

Tallon, M., \& Tallon-Bosc, I. 1992, A\&A, 253, 641 